\title{
Dynamical Theory of a Bidimensional System with an Infinite Number of Degrees of Freedom
}

\author{
O. de Pazzis ${ }^{\star}$ \\ Département de Physique, Observatoire de Meudon, Meudon, France
}

Received July 27, 1972

\begin{abstract}
The dynamical theory of a bidimensional model of hard squares with elastic collisions is presented. The time evolution is shown to exist on a large class of infinite configurations. Moreover, it is proved that any equilibrium state, that is any solution of the equilibrium equations, is concentrated on this set of allowed initial configurations and is invariant under the time evolution.
\end{abstract}

\section{§ 1. Introduction}

This paper is devoted to proving some results concerning the dynamical theory of a bidimensional system with infinitely many degrees of freedom. Namely we consider infinite configurations of particles that can be regarded as hard squares of a common size. As indicated in Fig. 1, these squares are restricted to having their sides parallel to the $x$ - and $y$-axis. Their velocities are all equal to $v_{0}$ in absolute values and parallel to any of the two bissectrix of $(O x, O y)$, so that there are only four possible velocities for each particle. The elastic collisions between the squares will be the only interactions considered.

Let us make some remarks to explain the motivation of the present work. The equilibrium theory of systems with infinitely many degrees of freedom has made many successes during the last decade, especially with regard to the interpretation of phase-transitions. It is tempting then to try to get some rigourous results in the domain of non-equilibrium theory. We quote here some typical problems which should be investigated on a rigourous basis:

1) Ergodic properties of thermodynamic equilibrium.

2) Existence of transport coefficients and their non-analytic behaviour at low density.

3) Irreversibility principle and $H$-theorem.

Unlike the third problem which lies in the field on non-equilibrium theory, the first two concern only dynamical properties in thermo-

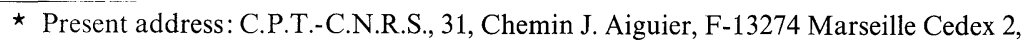
France. 
dynamic equilibrium. For problem 1) this is obvious, and for problem 2) this is suggested by Kubo's formula for transport coefficients.

In constructing the time evolution of configurations with infinitely many particles one is faced with a basic difficulty. In fact it is easy to see that for any realistic system some initial configurations can lead to catastrophic situations. The following example is well known: take a configuration of non interacting particles lying on the $x$-axis, with positions $q_{i}$ and momenta $p_{i}$ satisfying the relation $q_{i}=-p_{i}$. It is clear that at time one we shall have infinitely many particles at the origin, which is an unphysical situation.

Precisely the problem can be schematically formulated as follows. Let $[X]$ be the space of infinite configurations and $\varrho$ a probability measure on $[X]$. One has to find a subset $[\hat{X}] \subset[X]$ such that $\varrho[\hat{X}]=1$ and that the time evolution is defined on $[\hat{X}]$, namely $T^{t}([\hat{X}])=[\hat{X}]$. For such a measure $\varrho$, one can define $\varrho^{t}$ by the equation $\varrho^{t}(A)=\varrho\left(T^{-t} A\right)$ for any measurable set $A \subset[\hat{X}]$. In particular if $\varrho$ is an equilibrium state (in a sense to be precised) one expects that $\varrho$ is invariant under time evolution, that is $\varrho^{t}=\varrho$.

Such a programme has been carried out recently by Lanford [1,2], in the case of one-dimensional systems with bounded and finite range interactions. These results have been generalised to the case of a potential with hard-core by Sinai [3]. Moreover in the particular case of hard rods without any further interactions, Sinai has proved the ergodicity of the equilibrium state with respect to time evolution [3]. For a simple proof of this last property in the case of a semi-infinite system of hard rods see Ref. [4].

Unfortunately it is well known that one-dimensional systems exhibit properties which are often far from those of realistic systems. This is particularly clear concerning phase transitions. As for the dynamical properties, the validity of the one-dimensional Boltzmann equation, as a kinetic equation for long time scale, is seriously in doubt. It is then natural to extend the above results in higher dimensions. This extension is far from being immediate: the system of hard discs or hard spheres seems itself very intricate and remains an open problem. Here the difficulty lies essentially in the impossibility of controlling the particle velocities. This is the reason why we consider in this paper a rather simpler model with discrete velocities, all equal in absolute values, as described in the beginning of this introduction.

The main result of this paper can be stated in the following theorem:

Theorem. For any measure $\varrho_{z}$ on $[X]$ satisfying the equilibrium equations at activity $z$, there exists a Borel subset $[X]_{z}$ of $[X]$ such that $\varrho_{z}[X]_{z}=1$ and $T^{t}[X]_{z}=[X]_{z}$ for any value of $t$. Moreover $\varrho_{z}$ is invariant under $T^{t}$. 
In $\S 2$, we describe the space of infinite configurations $[X]$ for the model at hand and recall some known results about the equilibrium equations. Section 3 is devoted to the construction of a family of Borel subsets $[X]_{\alpha}^{0}$ of $[X]$ for which there exists a time evolution provided $|t|<\alpha$ is satisfied. In $\S 4$ we develop a straightforward but tedious process of simultaneous reduction of $[X]_{\alpha}^{0}$ and iteration of time evolution to get a family of Borel subsets $[X]_{\alpha}$ of $[X]$ such that $T^{t}[X]_{\alpha}{ }^{=}[X]_{\alpha}$ for any $t$. In Section 5 , we prove that for any measure $\varrho_{z}$ satisfying the equilibrium equations there exists an $[X]_{\alpha}$ of measure one with respect to $\varrho_{z}$, in addition we show the invariance of $\varrho_{z}$ under time evolution. This will complete the proof of the above theorem. We conclude with some remarks on the conservation of entropy and a straightforward extension of the results of this paper to a model of hard cubes in three dimensions.

\section{§ 2. Preliminaries}

In this section we define the space of infinite configurations for our model and quote some typical results which will be used in the rest of this paper. For more details the reader is referred to $[2,5,6]$.

The position of each square like particle (see Fig. 1) is given by the coordinates $q^{1}$ and $q^{2}$ of its center. The common mass and velocity are taken to be equal to one. The momentum $p$ of each particle is represented by one of the four numbers $\{1,2,3,4\}$ with the following convention: the momentum $j$ corresponds to the direction whose polar angle with the $x$-axis is given by $\pi / 4+(j-1) \pi / 2$.

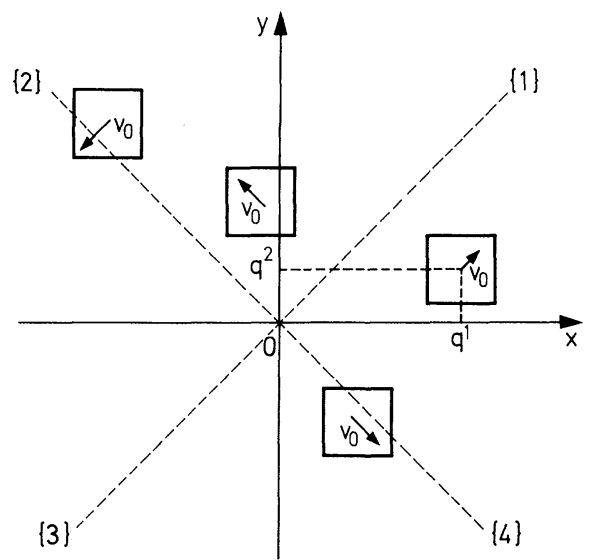

Fig. 1 
We call $X$ the space of all finite or infinite sequences $\left(q_{i}, p_{i}\right), i \in N$, with:

and

$$
\begin{gathered}
q_{i}=\left\{q_{i}^{1}, q_{i}^{2}\right\} \in R^{2} \\
p_{i} \in\{1,2,3,4\}=P
\end{gathered}
$$

$$
\left|q_{i}^{1}-q_{j}^{1}\right| \geqq a \text { or }\left|q_{i}^{2}-q_{j}^{2}\right| \geqq a \quad \text { if } \quad i \neq j,
$$

( $a$ is the common size of the squares).

We introduce the symmetry between the particles by the following equivalence relation. We say that two labelled configurations $X^{\prime}$ and $X^{\prime \prime}$ are equivalent iff they differ only by a permutation of the indexing set. $[X]$ will denote the space of equivalence classes and will be the "phasespace" for our model.

If $\Lambda$ is a subset of $R^{2}$ and $X \in[X]$, we denote by $X_{A}$ the configuration obtained from $X$ by considering only the particles whose center lies inside $\Lambda$. If $\Lambda$ is bounded $X_{A}$ is necessarily a finite configuration.

The space $[X]$ can be equipped with the following topology [2]. Let $\Omega^{1}$ be the space of all continuous mappings $f: R^{2} \times P \rightarrow R$, with compact supports. To each $f \in \mathfrak{R}^{1}$ corresponds a mapping $S f:[X] \rightarrow R$ :

$$
X=\left\{q_{i}, p_{i}\right\} \rightarrow S f(X)=\sum_{i} f\left(q_{i}, p_{i}\right) .
$$

We give $[X]$ the weakest topology making $S f$ continuous for every $f \in \mathfrak{K}^{1}$. We have the following property:

Proposition 2.1. $[X]$ with the above topology is a compact Polish space.

We give here a convenient criterion for the convergence of a sequence in $[X]$ :

Proposition 2.2. The sequence $X^{n}$ tends to $X \in[X]$ as $n \rightarrow \infty$ iff for every bounded open $\Lambda \subset R^{2}$ such that $X_{b d(\Lambda)}=\emptyset, X_{\Lambda}^{n}$ tends point by point to $X_{\Lambda}$.

We denote by $\mathfrak{U}$ the set of all continuous mappings $\varphi:[X] \rightarrow \mathbb{C}$, with its natural structure of $\mathbb{C}^{*}$-algebra. Then a state on $\mathfrak{U}$ is a positive Radon measure on $[X]$ of total mass one. A mapping $\varphi:[X] \rightarrow \mathbb{C}$ is said to be measurable in $\Lambda$, for $\Lambda \subset R^{2}$, iff $\varphi(X)=\varphi\left(X_{A}\right)$ for every $X \in[X]$. If $\Lambda$ is a bounded open of $R^{2}$, we set:

$$
\mathfrak{U}_{\Lambda}=\{\varphi \in \mathfrak{U} ; \quad \varphi \text { is measurable in } \Lambda\} .
$$

By the Stone-Weierstrass theorem we have:

Proposition 2.3. $\bigcup_{\Lambda} \mathfrak{H}_{\Lambda}$ is dense in $\mathfrak{u}$.

Proposition 2.3 shows that every state on $\mathfrak{U}$ is actually defined by its values on $\bigcup_{\Lambda} \mathfrak{H}_{\Lambda}$. This suggests to represent a state $\varrho$ on $\mathfrak{U}$ by a family 
of "local measures" $\varrho_{A}$ as follows [5]. If $\Lambda \subset R^{2}$ is a bounded open and non-empty set, we set:

$$
\left[X_{A}\right]=\left\{X_{A} ; X \in[X]\right\},
$$

$\left[X_{\Lambda}\right]$ can be identified with a subset of $\sum_{n \geqq 0}(\Lambda \times P)^{\hat{n}}$ where $(\Lambda \times P)^{\hat{n}}$ means the symmetrized product of $n$ copies of $\Lambda \times P$. If one gives $\sum_{n \geqq 0}(\Lambda \times P)^{n}$ its natural topology, then the mapping:

$$
\Pi_{\Lambda}:[X] \rightarrow \sum_{n \geqq 0}(\Lambda \times P)^{\hat{n}}: X \rightarrow X_{\Lambda}
$$

is a Borel mapping. Then if $\varrho$ is a state on $\mathfrak{U}, \varrho_{A}=\Pi_{\Lambda}(\varrho)$ will be a positive Radon measure on $\sum_{n \geqq 0}(\Lambda \times P)^{\hat{n}}$ of total mass one, with in addition $\varrho_{A}\left[X_{A}\right]=1$. It is easy to show that when $\Lambda$ runs over the bounded open subsets of $R^{2}$ the family $\varrho_{A}$ defines $\varrho$ in a unique way, provided some compatibility conditions are satisfied (see [5]). It is clear that if $\varphi \in \mathfrak{U}_{\Lambda}$ then:

$$
\int_{[X]} \varphi \varrho=\left.\int_{\left[X_{A}\right]} \varphi\right|_{\left[X_{A}\right]} \varrho_{A} .
$$

We conclude this section with a brief outline of the equilibrium equations $[6,7]$. We denote by $d X_{\Lambda}$ the measure on $\sum_{n \geqq 0}(\Lambda \times P)^{\hat{n}}$ defined
by:

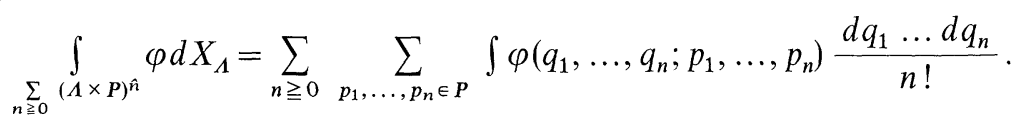

Every configuration $X \in[X]$ can be separated into two parts $X_{\Lambda}$ and $X_{R^{2} \backslash \Lambda}$. We say that a state $\varrho$ on $\mathfrak{U}$ satisfies the equilibrium equations at given $z \geqq 0$ iff for every bounded open $\Lambda \subset R^{2}$ and $\varphi \in \mathfrak{U}$ :

$$
\int_{[X]} \varphi \varrho=\int_{\left[X_{A}\right]} d X_{\Lambda}(z / 4)^{N\left(X_{A}\right)} \int_{\left[X_{R^{2} \backslash A}\right]} f\left(X_{\Lambda} \mid Y_{R^{2} \backslash A}\right) \varphi\left(X_{\Lambda}+Y_{R^{2} \backslash A}\right) \varrho\left(Y_{R^{2} \backslash A}\right)
$$

where $N\left(X_{A}\right)$ is the number of particles of $X_{A}$ and

$$
f\left(X_{\Lambda} \mid Y_{R^{2} \backslash \Lambda}\right)= \begin{cases}1 & \text { if } X_{\Lambda}+Y_{R^{2} \backslash \Lambda} \in[X] \\ 0 & \text { otherwise }\end{cases}
$$

(the factor 4 comes out from the normalisation in momentum space).

It can be proved that for $z$ sufficiently small, in particular for $z<1 / e a^{2}$, there is a unique state satisfying (2.2) and this state is obtained from the grandcanonical ensemble by taking the thermodynamic limit. In view of (2.1) and (2.2) we see that if a state $\varrho$ satisfies (2.2) its local measures $\varrho_{A}$ are absolutely continuous with respect to $d X_{A}$ and:

$$
\varrho_{\Lambda}=(z / 4)^{N\left(X_{\Lambda}\right)} d X_{\Lambda} \int_{\left[X_{R^{2} \backslash \backslash}\right]} f\left(X_{\Lambda} \mid Y_{R^{2} \backslash \Lambda}\right) \varrho\left(Y_{R^{2} \backslash \Lambda}\right) .
$$




\section{§ 3. Time Evolution of Infinite Configurations}

In this section we shall exhibit a class of initial configurations for which there exists a time evolution provided the amplitude of the time interval is sufficiently small. First we will need some classical results on the dynamics of finite configurations in boxes.

\subsection{Time Evolution of Finite Configurations}

In the rest of this paper $\Lambda$ will denote any rectangular box with sides parallel to the $x$ - and $y$-axis, $A+a$ will be the rectangular box obtained from $\Lambda$ by adding a to its sides and with the same center as $\Lambda$. Finally $\Lambda_{l}$ with $l>0$ means the square box centered at the origine and whose area is $l^{2}$.

Take now a finite configuration $X \in\left[X_{A}\right]$. We may consider $X$ as enclosed in the rectangular and rigid box $\Lambda+a$ and take the time evolution as follows: we suppose that the squares suffer elastic collisions with each other and with the boundary of the box $\Lambda+a$. In Fig. 2 we have indicated the relative motion of two squares during each of the three possibilities of collisions between them. We emphasize that the assumption that all the particles have the same mass and velocity is essential in order to preserve the discrete character of the momentum space during a collision.

If we call $T_{A}^{t}(X)$ the evolution at time $t$ of the initial configuration $X \in\left[X_{A}\right]$, it appears that $T_{A}^{t}$ is not a one to one mapping on $\left[X_{A}\right]$. This slight complication is always present when considering instantaneous collisions. Let $\left[X_{A}\right]^{-}$be the subset of configurations $X \in\left[X_{A}\right]$ such that there is at least one particle of $X$ which "enters" a collision with another

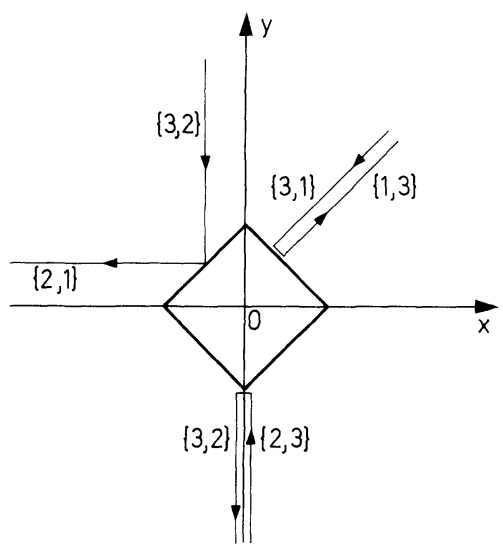

Fig. 2 
one or with the boundary of $A+a$. Clearly $\left[X_{A}\right]^{-}$is a closed and therefore Borel subset of $\left[X_{A}\right]$. If we disregard the configurations belonging to $\left[X_{A}\right]^{-}$we may state the following classical result:

Proposition 3.1. a) $T_{A}^{t}: R \times\left[X_{A}\right] \rightarrow\left[X_{A}\right] \backslash\left[X_{A}\right]^{-}: X \rightarrow T_{A}^{t}(X)$ is a Borel mapping.

b) $T_{A}^{t}: R \times\left[X_{A}\right] \backslash\left[X_{A}\right]^{-} \rightarrow\left[X_{A}\right] \backslash\left[X_{A}\right]^{-}$is a one-parameter group of Borel isomorphisms.

c) $\left[X_{A}\right]^{-}$is a null set with respect to $d X_{A}$ and $d X_{A}$ is invariant under $T_{A}^{t}$.

\subsection{Thermodynamic Limit of $T_{A}^{t}$}

We may now precise what we mean by $T^{t}(X)$ when $X$ is an infinite configuration in $[X]$. For each rectangular box $\Lambda$ we may consider $T_{A}^{t}\left(X_{A}\right)$ as a finite configuration in $[X]$ and take the limit, on the net of increasing boxes, of $T_{A}^{t}\left(X_{A}\right)$ in the topology of $[X]$. If this limit exists we define it to be $T^{t}(X)$. We introduce, the natural domain of definition of $T^{t}$ :

$$
\mathscr{D}=\left\{(t, X) \in R \times[X] ; \lim _{\Lambda \rightarrow \infty} T_{\Lambda}^{t}\left(X_{\Lambda}\right)=T^{t}(X) \text { exists }\right\}
$$

We shall use throughout this paper the following criterion for the existence of $T^{t}(X)$ :

Proposition 3.2. Let $(t, X) \in R \times[X]$, if for every bounded open $\Lambda^{\prime} \subset R^{2}$ there exists a rectangular box $\Lambda^{0}$ such that:

$$
\left(T_{\Lambda}^{t}\left(X_{\Lambda}\right)\right)_{\Lambda^{\prime}}=\left(T_{\Lambda^{0}}^{t}\left(X_{\Lambda^{0}}\right)\right)_{\Lambda^{\prime}} \quad \forall \Lambda \supset \Lambda^{0}
$$

then $(t, X) \in \mathscr{D}$ and $\left(T^{t}(X)\right)_{\Lambda^{\prime}}=\left(T_{\Lambda}^{t}\left(X_{\Lambda}\right)\right)_{\Lambda^{\prime}} \forall \Lambda \supset \Lambda^{0}$.

This proposition follows at once from Proposition 2.2. It means the simple fact that if $(t, X)$ satisfies the conditions of the proposition, the situation at time $t$ in any bounded subset of $R^{2}$ is given by the evolution in a sufficiently large box. We denote by $\mathscr{D}^{\prime}$ the subset of $\mathscr{D}$ for which the conditions of the above proposition are satisfied.

Before going further we introduce the following definition: let $\alpha>0$, $I_{\alpha}=(-\alpha,+\alpha)$ and $A \subset[X]$, we say that $T^{t}$ is a local one-parameter group on $I_{\alpha} \times A$ iff:

(D1) $I_{\alpha} \times A \subset \mathscr{D}^{\prime}$.

(D2) $\forall X \in A, \forall t_{1}, t_{2} ;\left|t_{1}\right|+\left|t_{2}\right|<\alpha:\left(t_{2}, T^{t_{1}}(X)\right) \in \mathscr{D}^{\prime}$ and $T^{t_{2}}\left(T^{t_{1}}(X)\right)$ $=T^{t_{2}+t_{1}}(X)$.

\subsection{A Class of Initial Configurations}

The aim of this paragraph is the construction of a class of initial configurations $[X]_{\alpha}^{0}$ such that $I_{\alpha} \times[X]_{\alpha}^{0}$ belongs to $\mathscr{D}^{\prime}$. To this purpose 
we introduce the following notations: let $\alpha>0$ and $X \in[X],\left\{q_{i}, p_{i}\right\}$ a representative of $X$, we say that particles $i$ and $j$ of $X$ are $\alpha$-adjacent iff:

and

$$
\left|q_{i}^{1}-q_{j}^{1}\right|<a+\alpha \cdot \sqrt{2}
$$

$$
\left|q_{i}^{2}-q_{j}^{2}\right|<a+\alpha \cdot \sqrt{2} \text {. }
$$

A part $X^{\prime}$ of the configuration $X$ is said an $\alpha$-chain of $X$ if there exists a representative $X^{\prime}=\left\{q_{i}, p_{i}\right\}$ of $X^{\prime}$ such that particles $i$ and $i+1$ are $\alpha$-adjacent for every $i$. Let $X^{\prime}$ and $X^{\prime \prime}$ be two disjoint parts of $X$, we say that $X^{\prime}$ and $X^{\prime \prime}$ are $\alpha$-disconnected if there does not exist an $\alpha$-chain of $X^{\prime}+X^{\prime \prime}$ composed with particles from both $X^{\prime}$ and $X^{\prime \prime}$. If $X^{\prime}$ is a finite $\alpha$-chain, the particle number of $X^{\prime}$ is called the "length" of $X^{\prime}$.

Consider now the square box $\Lambda_{l}$ with $l>0$ (see $\left.\S 3.1\right)$. For $X \in[X], X_{\Lambda_{l}}$ is a finite configuration. We denote by $\left|X_{\Lambda_{l}}\right|_{\alpha}$ the length of the longest $\alpha$-chain of $X_{\Lambda_{l}}$. We set:

$$
[X]_{\alpha}^{\prime}=\left\{X \in[X] ; \sup _{l>0} \frac{\left|X_{\Lambda_{l}}\right|_{\alpha}}{\log _{+} l}<+\infty\right\}
$$

where $\log _{+} l=\log (\sup (l, e))$. In the following it will be convenient to consider the subsets of $[X]_{\alpha}^{\prime}$ :

$$
[X]_{\alpha, K}^{\prime}=\left\{X \in[X] ; \sup _{l>0} \frac{\left|X_{\Lambda_{l}}\right|_{\alpha}}{\log _{+} l} \leqq K\right\} .
$$

Finally, for the same reasons as in $\S 3.1$, we are led to disregard the subset of configurations having at least two particles "entering" a collision:

We set:

$$
[X]^{-}=\left\{\begin{array}{c}
X \in[X] ; \\
\text { "there are at least two particles of } X \\
\text { "entering" a collison }
\end{array}\right\} .
$$

$$
[X]_{\alpha}^{\prime \prime}=[X]_{\alpha}^{\prime} \cap\left([X] \backslash[X]^{-}\right) .
$$

Proposition 3.3. $[X]_{\alpha}^{\prime \prime}$ is a Borel subset of $[X]$.

Proof. First we show that $[X]^{-}$is a Borel set. To this purpose we choose an increasing sequence of square boxes. $\Lambda_{n}$ with $n \in N$; we remark that the subset of configurations of $\left[X_{\Lambda_{n}}\right]$ having at least two particles "entering" a collision is closed in $\left[X_{A_{n}}\right]$, then the inverse image through $\Pi_{A_{n}}$ of this subset is a Borel set in $[X]$. Therefore $[X]^{-}$is a Borel set as being a countable union of Borel sets.

It remains to prove that $[X]_{\alpha}^{\prime}$ is a Borel set. But it follows from Proposition 2.2 that $[X]_{\alpha, K}^{\prime}$ is closed in $[X]$; then choose an increasing sequence $K_{n} \rightarrow+\infty$ and the assertion is proved because $[X]_{\alpha}^{\prime}=\bigcup_{n}[X]_{\alpha, K_{n}}^{\prime}$ 


\subsection{An Existence Theorem}

Here we prove the existence of a time evolution for initial configurations in $[X]_{\alpha}^{\prime}$ provided the amplitude of the time interval is sufficiently small.

Proposition 3.4. a) $\forall \alpha>0, I_{\alpha} \times[X]_{\alpha}^{\prime} \subset \mathscr{D}^{\prime}$.

b) $\forall \alpha>0, \forall \Lambda \subset R^{2}$, bounded open, $\forall K>0$, $\exists l_{0}:\left(T^{t}(X)\right)_{\Lambda}=\left(T_{\Lambda^{\prime \prime}}^{t}\left(X_{\Lambda^{\prime \prime}}\right)\right)_{\Lambda} ; \quad \forall \Lambda^{\prime \prime} \supset \Lambda_{l_{0}}, \quad \forall X \in[X]_{\alpha, K}^{\prime}, \quad \forall t, \quad|t|<\alpha$.

c) $T^{t}: I_{\alpha} \times[X]_{\alpha}^{\prime} \rightarrow[X] \backslash[X]^{-}$is a Borel mapping.

d) $T^{t}$ is a local one-parameter group on $I_{x} \times[X]_{\alpha}^{\prime \prime}$.

Proof. a) Is a consequence of $b$ ), hence we prove b). Knowing that each particle has a velocity with modulus one, we see that the particles which enter in $\Lambda$ during the time interval $(-\alpha,+\alpha)$ are necessarily at time zero in some square box $\Lambda_{l^{\prime}}, l^{\prime}$ depending only on $\alpha$ and $\Lambda$. Let us choose $l_{0}$ such that if $l>l_{0}$ then $l-l^{\prime}>2 K(\log l)(a+\alpha \sqrt{2})$. This implies that $X_{\Lambda^{\prime}}$ is $\alpha$-disconnected from $X_{R^{2} \backslash A^{\prime \prime}}$ for any $X \in[X]_{\alpha, K}^{\prime}$ and any $\Lambda^{\prime \prime} \supset \Lambda_{l_{0}}$ (see Fig. 3). That is, the motion of the particles of $X$ initially in $\Lambda_{l^{\prime}}$ is not perturbed by the particles of $X$ initially outside $\Lambda_{l_{0}}$ so that:

$\forall \Lambda^{\prime \prime} \supset \Lambda_{l_{0}}, \forall t,|t|<\alpha, \forall X \in[X]_{\alpha, K}^{\prime}$ :

$$
\left(T_{\Lambda^{\prime \prime}}^{t}\left(X_{\Lambda^{\prime \prime}}\right)\right)_{\Lambda}=\left(T_{\Lambda_{l_{0}}}^{t}\left(X_{\Lambda_{l_{0}}}\right)\right)_{\Lambda}=\left(T^{t}(X)\right)_{\Lambda} .
$$

To prove c) we note that if $(t, X) \in I_{\alpha} \times[X]_{\alpha}^{\prime}$ then

$$
T^{t}(X)=\lim _{l \rightarrow \infty} \cdot i_{\Lambda_{l}} \circ T_{\Lambda_{l}}^{t} \circ \Pi_{\Lambda_{l}}(X)
$$

where $i_{\Lambda_{l}}$ is the inclusion: $[X]_{\Lambda_{l}} \rightarrow[X]$ which is a Borel mapping, $T_{\Lambda_{l}}^{t}$ is a Borel mapping from Proposition 3.1 and $\Pi_{A_{l}}$ is a Borel mapping so

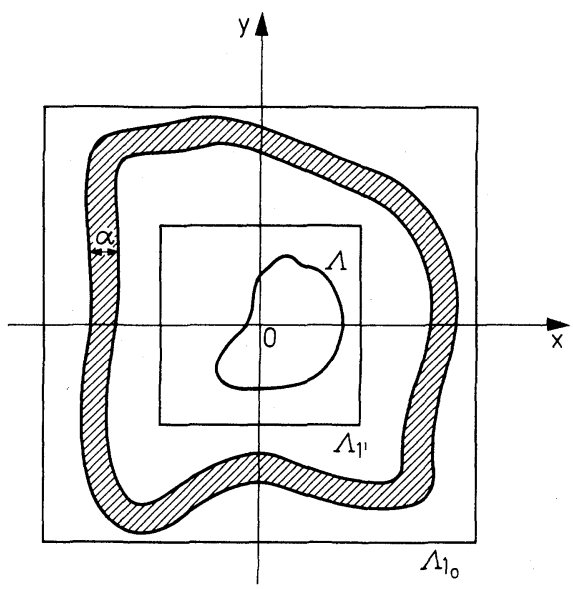

Fig. 3 
that $i_{\Lambda_{l}} \circ T_{\Lambda_{l}}^{t} \circ \Pi_{\Lambda_{l}}$ is a Borel mapping too. Therefore $T^{t}(X)$ is a Borel mapping as being the pointwise limit of a family of Borel mappings with values in a metric space.

It remains to prove d). Let $X \in[X]_{\alpha}^{\prime \prime}$ and $t_{1}, t_{2}$ such that $\left|t_{1}\right|+\left|t_{2}\right|<\alpha$, it is clear that $T^{t_{1}}(X) \in[X]_{\alpha-\left|t_{1}\right|}^{\prime \prime}$ so that $\left(t_{2}, T^{t_{1}}(X)\right) \in \mathscr{D}^{\prime}$. On the other hand, from $b$ ) we have for any bounded open $A$ :

$$
\left(T^{t_{2}+t_{1}}(X)\right)_{\Lambda}=\left(T_{\Lambda_{l_{0}}}^{t_{2}+t_{1}}\left(X_{\Lambda_{l_{0}}}\right)\right)_{\Lambda}=\left(T_{\Lambda_{l_{0}}}^{t_{2}}\left(T_{\Lambda_{l_{0}}}^{t_{1}}\left(X_{\Lambda_{l_{0}}}\right)\right)\right)_{\Lambda} .
$$

With the same argument as used in the proof of b), we have:

$$
\left(T_{\Lambda_{l_{0}}}^{t_{2}}\left(T_{\Lambda_{l}}^{t_{1}}\left(X_{\Lambda_{l}}\right)\right)\right)=\left(T^{t_{2}}\left(T^{t_{1}}(X)\right)\right)_{\Lambda},
$$

so that $\left(T^{t_{2}+t_{1}}(X)\right)_{\Lambda}=\left(T^{t_{2}}\left(T^{t_{1}}(X)\right)\right)_{\Lambda}$ for any bounded open $\Lambda$. Hence the assertion is proved.

In $\S 5$ we shall need a slight modification of the preceding result for proving the time invariance of the equilibrium states. We modify the boundary of the box $\Lambda_{l+a}$ in the following way: given a configuration $X \in[X]$ and a box $\Lambda_{l}$, we freeze all the particles of $X$ whose center lies in $\Lambda_{l+2 a} \backslash \Lambda_{l}$ and we consider the motion of the particles of $X_{A_{l}}$ taking into account elastic collisions on both the boundary of $\Lambda_{l+a}$ and the frozen particles in $\Lambda_{l+2 a} \backslash \Lambda_{l}$. This time evolution is denoted by $\tilde{T}_{\Lambda_{l}}^{t}\left(X_{\Lambda_{l+2 a}}\right)$ (see Fig. 4). The same argument as used in the proof of Proposition 3.4b) leads to the following result:

Proposition 3.5. $\forall \alpha>0, \forall A \subset R^{2}$ bounded open, $\forall K>0$,

$$
\exists l:\left(T^{t}(X)\right)_{\Lambda}=\left(\tilde{T}_{\Lambda_{l}}^{t}\left(X_{A_{l+2 a}}\right)\right)_{\Lambda}, \quad \forall X \in[X]_{\alpha, K}^{\prime}, \quad \forall t, \quad|t|<\alpha .
$$

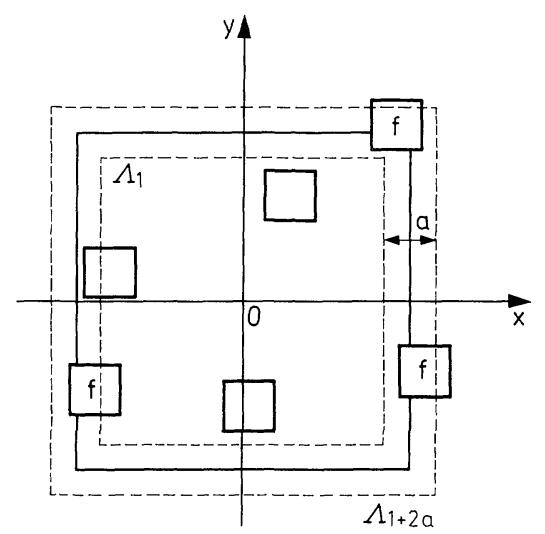

Fig. 4 


\section{§ 4. Iteration of the Time Evolution}

We proved in the preceding section the existence of a local time evolution on a Borel set $[X]_{\alpha}^{\prime \prime}$ of initial configurations. But we do not have the time invariance of $[X]_{\alpha}^{\prime \prime}$, that is: $T^{t}[X]_{\alpha}^{\prime \prime} \neq[X]_{\alpha}^{\prime \prime}$ if $|t|<\alpha$, so that we cannot iterate directly the time evolution. The aim of this paragraph is precisely to perform a simultaneous reduction of the initial class $[X]_{\alpha}^{\prime \prime}$ and iteration of the time evolution to get a Borel set $[X]_{\alpha}$ such that $T^{t}[X]_{\alpha}=[X]_{\alpha}, \forall t \in R$.

First we consider: $[X]_{\alpha}^{0}=\bigcap_{0<\beta<\alpha}[X]_{\beta}^{\prime \prime}$. As $[X]_{\beta_{1}}^{\prime \prime} \subset[X]_{\beta_{2}}^{\prime \prime}$ if $\beta_{1}>\beta_{2}$, it is clear that $[X]_{\alpha}^{\prime \prime} \subset[X]_{\alpha}^{0}$. On the other hand, $[X]_{\alpha}^{0}=\bigcap_{0<\beta_{n}<\alpha}[X]_{\beta_{n}}^{\prime \prime}$ for any monotonically increasing sequence $\beta_{n}$ such that $\lim _{n \rightarrow \infty} \beta_{n}=\alpha$. This implies, in particular, that $[X]_{\alpha}^{0}$ is a Borel subset of $[X]$. (It can be noted that $[X]_{\alpha}^{\prime \prime}$ is a proper subset of $[X]_{\alpha}^{0}$.) It is clear also that the statements a), c) and d) of Proposition 3.4 still apply on the class of initial configurations $[X]_{\alpha}^{0}$. Now we reduce $[X]_{\alpha}^{0}$ as follows: we consider the sequence:

$$
\begin{aligned}
& {[X]_{\alpha}^{1}=\bigcap_{\substack{t \in Q \\
|t|<\alpha / 2}} T^{t}[X]_{\alpha}^{0}} \\
& \ldots \ldots \ldots \ldots \ldots \ldots \ldots \ldots \ldots \ldots \ldots \ldots \\
& {[X]_{\alpha}^{n}=\bigcap_{\substack{t \in Q \\
|t|<\alpha / 2}} T^{t}[X]_{\alpha}^{n-1}}
\end{aligned}
$$

where $Q$ means the set of rational numbers. From Proposition 3.4 it follows that $[X]_{\alpha}^{n}$ is a Borel set for every $n$, and:

$$
\cdots \subset[X]_{\alpha}^{n} \subset[X]_{\alpha}^{n-1} \subset \cdots \subset[X]_{\alpha}^{0} .
$$

Proposition 4.1. $T^{t}$ is a local one-parameter group on $I_{n \alpha / 2} \times[X]_{\alpha}^{n}$.

First we prove the following lemma:

Lemma 4.1. If $T^{t}$ is a local one-parameter group on $I_{t_{0}} \times\{X\}$ and $T^{t}(X) \in[X]_{\alpha}^{0}$ for every $t \in I_{t_{0}}$, then $T^{t}$ is a one-parameter group on $I_{t_{0}+\alpha} \times\{X\}$.

Proof. First we prove that $(t, X) \in \mathscr{D}^{\prime}$ when $t_{0} \leqq|t|<t_{0}+\alpha$. We may assume $t>0$ for example. Then there exist $t_{1}, t_{2}$ such that $t=t_{1}+t_{2}$ and $0<t_{1}<t_{0}, 0<t_{2}<\alpha$. We may choose some $\beta$ such that $t_{2}<\beta<\alpha$. From the assumption of the lemma we get:

$$
\left(t_{1}, X\right) \in \mathscr{D}^{\prime} \text { and } T^{t_{1}}(X) \in[X]_{\beta, K}^{\prime} \text { for some } K>0 .
$$

Now we consider a bounded open $\Lambda \subset R^{2}$. The particles of $T^{t_{1}}(X)$ which move in $\Lambda$ during the time interval $\left(t_{1}, t\right]$ are necessarily in some square box $\Lambda_{l_{1}}$ at time $t_{1}$. As $T^{t_{1}}(X) \in[X]_{\beta, K}^{\prime}$, there exists $l_{2}>0$ such that 
$\left(T^{t_{1}}(X)\right)_{\Lambda_{l_{1}}}$ is $\beta$-disconnected from $\left(T^{t_{1}}(X)\right)_{R^{2} \backslash \Lambda_{l_{2}}}$. Finally as $\left(t_{1}, X\right) \in \mathscr{D}^{\prime}$ there exists $l_{3}>0$ such that:

Therefore:

$$
\forall \Lambda^{\prime} \supset \Lambda_{l_{3}}:\left(T_{\Lambda^{\prime}}^{t_{1}}\left(X_{\Lambda^{\prime}}\right)\right)_{\Lambda_{l_{2}}}=\left(T^{t_{1}}(X)\right)_{\Lambda_{l_{2}}} .
$$

$$
\begin{aligned}
\exists l_{3}>0: \forall \Lambda^{\prime} \supset \Lambda_{l_{3}}:\left(T_{\Lambda^{\prime}}^{t_{1}+t_{2}}\left(X_{\Lambda^{\prime}}\right)\right)_{\Lambda} & =\left(T_{\Lambda^{\prime}}^{t_{2}}\left(T_{\Lambda^{\prime}}^{t_{1}}\left(X_{\Lambda^{\prime}}\right)\right)\right)_{\Lambda} \\
& =\left(T_{\Lambda^{\prime}}^{t_{2}}\left(T_{\Lambda^{\prime}}^{t_{1}}\left(X_{\Lambda^{\prime}}\right)_{\Lambda_{l_{2}}}\right)\right)_{\Lambda} \text { as } t_{2}<\beta \\
& =\left(T_{\Lambda^{\prime}}^{t_{2}}\left(T^{t_{1}}(X)_{\Lambda_{l_{2}}}\right)\right)_{\Lambda} \text { from } \\
& =\left(T^{t_{2}}\left(T^{t_{1}}(X)\right)\right)_{\Lambda} \text { because }
\end{aligned}
$$

$\left(T^{t_{1}}(X)\right)_{\Lambda_{l_{1}}}$ is $\beta$-disconnected from $\left(T^{t_{1}}(X)\right)_{R^{2} \backslash \Lambda_{l_{2}}}$. Then we have proved that $(t, X) \in \mathscr{D}^{\prime}$ if $t_{0} \leqq|t|<t_{0}+\alpha$ with in addition:

$$
T^{t}(X)=T^{t_{2}}\left(T^{t_{1}}(X)\right) \quad \text { if } \quad t=t_{1}+t_{2} \quad \text { and } \quad\left|t_{1}\right|<t_{0}, \quad\left|t_{2}\right|<\alpha .
$$

To get the local one-parameter group property we remark that if $t=t_{1}+t_{2}$ with $\left|t_{1}\right|+\left|t_{2}\right|<t_{0}+\alpha$ it is always possible to write: $t=t_{1}^{\prime}+t_{1}^{\prime \prime}+t_{2}$ with $\left|t_{1}^{\prime \prime}\right|+\left|t_{2}\right|<t_{0},\left|t_{1}^{\prime}\right|<\alpha$. It suffices then to apply twice (4.5) to prove the assertion.

We turn now to the prove of the proposition. For $n=1$ we have $[X]_{\alpha}^{1} \subset[X]_{\alpha}^{0}$ so that the property is a consequence of Proposition 3.4. By induction we assume that the property holds for $n-1$, and we consider $X \in[X]_{\alpha}^{n}$. We have $[X]_{\alpha}^{n} \subset[X]_{\alpha}^{n-1}$ so that $T^{t}$ is a local one-parameter group on $I_{(n-1) \alpha / 2} \times\{X\}$. Moreover $T^{t}(X) \in[X]_{\alpha}^{0}$ for every $t \in Q$ and $|t|<(n-1) \alpha / 2$. We show that this property still holds for $t \in R$. Indeed let $t \in R$ with $|t|<(n-1) \alpha / 2$. We can choose a monotonically increasing sequence of rational numbers $t_{n}$ such that $\lim _{n \rightarrow \infty} t_{n}=t$. We have from the assumption:

$$
T^{t_{n}}(X) \in[X]_{\alpha}^{0}=\left([X] \backslash[X]^{-}\right) \bigcap_{0<\beta<\alpha}[X]_{\beta}^{\prime}
$$

we already know that $T^{t}(X) \in[X] \backslash[X]^{-}$by the very definition of $T^{t}$. To prove that $T^{t}(X) \in \bigcap_{0<\beta<\alpha}[X]_{\alpha}^{\prime}$ we remark that $T^{t_{n}}(X) \in[X]_{\alpha-\left(t-t_{n}\right)}^{\prime}$ for $n$ sufficiently large, as a consequence of (4.6). Then $T^{t}(X) \in[X]_{\alpha-3\left(t-t_{n}\right)}^{\prime}$ for $n$ sufficiently large, because all particles have a velocity with modulus one. Therefore:

$$
T^{t}(X) \in \bigcap_{n \geqq n_{0}}[X]_{\alpha-3\left(t-t_{n}\right)}^{\prime}=\bigcap_{0<\beta<\alpha}[X]_{\beta}^{\prime}
$$

as $\lim _{n \rightarrow \infty}\left(t-t_{n}\right)=0$. Hence we have $T^{t}(X) \in[X]_{\alpha}^{0}$ for every real $t$ such that $|t|<(n-1) \alpha^{\prime} 2$. We apply Lemma 4.2 to assert that $T^{t}$ is a local oneparameter group on $I_{n \alpha / 2} \times\{X\}$ every $X \in[X]_{\alpha}^{n}$. This achieves the proof of the proposition.

We set $[X]_{\alpha}=\bigcap_{n \geqq 0}[X]_{\alpha}^{n}$, then we have the final result: 
Proposition 4.2. a) $[X]_{\alpha}$ is a Borel set of $[X]$,

b) $R \times[X]_{\alpha} \subset \mathscr{D}^{\prime}$,

c) $T^{t}: R \times[X]_{\alpha} \rightarrow[X]_{\alpha}$ is a Borel mapping and forms a one parameter group of Borel isomorphisms on $[X]_{\alpha}$.

Proof. a) Is obvious, b) follows immediately from Proposition 4.1. For c), it sufficies to prove that $T^{t}[X]_{\alpha} \subset[X]_{\alpha}$, then the rest will follow from Proposition 4.1. Now the property $T^{t}[X]_{\alpha} \subset[X]_{\alpha}$ is an easy consequence of the following lemma:

\section{Lemma 4.2. $T^{t}[X]_{\alpha}^{n} \subset[X]_{\alpha}^{n-1}$ if $|t|<\alpha / 2$.}

Proof. The property is true for $t \in Q,|t|<\alpha / 2$ by the very definition of $[X]_{\alpha}^{n}$. We suppose now $t \in R$ and $|t|<\alpha / 2$. We have to prove that for any rational $t_{1}$ such that $\left|t_{1}\right|<(n-1) \alpha / 2 T^{t_{1}}\left(T^{t}(X)\right) \in[X]_{\alpha}^{0}$ if $X \in[X]_{\alpha}^{n}$. But from Proposition 4.1, we have:

$$
T^{t_{1}}\left(T^{t}(X)\right)=T^{t_{1}+t}(X)=T^{t+t_{1}}(X)=T^{t}\left(T^{t_{1}}(X)\right) \quad \text { with } \quad T^{t_{1}}(X) \in[X]_{\alpha}^{1}
$$

so that: $T^{t^{\prime}}\left(T^{t_{1}}(X)\right) \in[X]_{\alpha}^{0}$ for every $t^{\prime} \in Q,\left|t^{\prime}\right|<\alpha / 2$. With the same argument as used in the proof of Proposition 4.1, we have: $T^{t}\left(T^{t_{1}}(X)\right) \in[X]_{\alpha}^{0}$ for every $t \in R, \mid t \|<\alpha / 2$. This proves the lemma and therefore the proposition.

Before applying the above results to statistical mechanics, we make a remark concerning the commutativity of $T^{t}$ with the group of natural displacements acting on $[X]$. In fact, if we call $G$ the subgroup of displacements of $R^{2}$ composed with the translations and the rotations of angle $k \pi / 2$ with $k \in Z, G$ acts in a natural way on $[X]$. Then it is easy to prove the following proposition:

Proposition 4.3. $\forall \alpha>0,[X]_{\alpha}$ is invariant under $G$ and:

$$
T^{t} \circ G=G \circ T^{t} .
$$

\section{§ 5. Time Evolution of States}

The previous Proposition 4.2 ensures the existence of a class of configurations for which we have a satisfactory time evolution. Consider now a state $\varrho$ on $\mathfrak{U}$ and suppose that for some $\alpha>0, \varrho[X]_{\alpha}=1$, then we can define a state $\varrho^{t}$ on $[X]$ by:

$$
\int \varphi \varrho^{t}=\int\left(\varphi \circ T^{t}\right) \varrho \quad \text { for any } \quad \varphi \in \mathfrak{U} .
$$

Indeed it is easy to see that (5.1) defines a state on $[X]$. Hence for such a state we have a time evolution.

Consequently, the usefulness of Proposition 4.2 will depend on wether or not interesting states with respect to statistical mechanics are concentrated on $[X]_{\alpha}$ for some $\alpha>0$. We shall be satisfied with proving that any solution of the equilibrium equations (2.2) is a state concentrated 
on $[X]_{\alpha}$ for some $\alpha$ sufficiently small. In addition we shall prove the time invariance of such a state.

We give here an outline of the argument:

a) For $z$ given in (2.2), we prove that there exists $\alpha(z)>0$ such that any solution $\varrho_{z}$ of $(2.2)$ is concentrated on $[X]_{\alpha}^{\prime \prime}$ namely $\varrho_{z}[X]_{\alpha}^{\prime \prime}=1$, $\forall \alpha \leqq \alpha(z)$.

b) For such a $\varrho_{z}$ we can define $\varrho_{z}^{t}$ provided $|t|<\alpha(z)$. Then we prove that $\varrho_{z}^{t}=\varrho^{t}$ if $|t|<\alpha(z)$.

c) This will imply that $\varrho_{z}$ is concentrated on $[X]_{\alpha}$ if $\alpha \leqq \alpha(z)$ and $\varrho_{z}$ is invariant under $T^{t}$.

First we introduce some notations that will be used in the following. Let $p \in N$ be a given integer, we consider the horizontals and verticals of abscissas and ordinates $m a / p$ with $m \in Z$, which separate the plane $R^{2}$ into cells of area $(a / p)^{2}$. The cell limited by the horizontals $m a / p,(m+1) a / p$ and the verticals $n a / p,(n+1) a / p$ will be denoted by the pair $(m, n)$. We say that two cells $\left(m_{i}, n_{i}\right)$ and $\left(m_{j}, n_{j}\right)$ are $p$-adjacent iff:

$$
\left|m_{i}-m_{j}\right|=p \quad \text { and } \quad\left|n_{i}-n_{j}\right| \leqq p \quad \text { ore vice-versa } .
$$

As in $\S 3.3$, a $p$-chain of cells will be a sequence $\left(m_{i}, n_{i}\right)$ such that $\left(m_{j}, n_{j}\right)$ and $\left(m_{j+1}, n_{j+1}\right)$ are $p$-adjacent for every $j$.

If $X \in[X]$ and $C$ is a $p$-chain of cells, we say that $C$ is occupied by $X$ if every cell of $C$ is occupied by a particle of $X$. It will be convenient to set:

$$
[C]=\{X \in[X] ; C \text { is occupied by } X\} \text {. }
$$

Finally if $\Lambda_{2 N a / p}$ is a square box, we denote by $C_{N, r}^{p}$ the set of all $p$-chains of cells, of length $r$, in the box $\Lambda_{2 N a / p}$. We now prove the first step:

Proposition 5.1. If $\varrho_{z}$ is a solution of (2.2) there exists $\alpha(z)$ such that $\varrho_{z}[X]_{\alpha}^{\prime \prime}=1$ for every $\alpha \leqq \alpha(z)$.

Proof. $[X]_{\alpha}^{\prime \prime}=\left([X] \backslash[X]^{-}\right) \cap[X]_{\alpha}^{\prime}$ we first prove that $\varrho_{z}\left([X] \backslash[X]^{-}\right)=1$, that is $\varrho_{z}[X]^{-}=0$. We choose an encreasing sequence of square boxes $\Lambda_{n}$ with $n \in N$, we remark that the subset of configurations having at least two particles "entering" a collision in $\Lambda_{n}$ is a null set with respect to $d X_{\Lambda_{n}}$, therefore with respect to $\varrho_{z}$, as $\left(\varrho_{z}\right)_{\Lambda_{n}}$ is absolutely continuous with respect to $d X_{A_{n}}$ (see 2.3). Then $[X]^{-}$is a null set, as being a countable union of null sets. We now prove that $\varrho_{z}[X]_{\alpha}^{\prime}=1$ for $\alpha$ sufficiently small. This is contained in the following lemma:

Lemma 5.2. There exists $\alpha(z)>0$ such that if $\varrho_{z}$ is a solution of (2.2):

$$
\lim _{K \rightarrow \infty} \varrho_{z}\left([X] \backslash[X]_{\alpha, K}^{\prime}\right)=0, \forall \alpha \leqq \alpha(z) \text {. }
$$

Proof. As $[X]_{\alpha_{1}, K}^{\prime} \subset[X]_{\alpha_{2}, K}^{\prime}$ if $\alpha_{2}<\alpha_{1}$ it sufficies to prove the existence of an $\alpha(z)>0$ such that:

$$
\lim _{K \rightarrow \infty} \varrho_{z}\left([X]^{\prime}[X]_{\alpha(z), K}^{\prime}\right)=0 .
$$


Moreover we may seek an $\alpha(z)$ such that $\alpha(z) \cdot \sqrt{2}=a / p$ where $p$ is an integer. Therefore if we set:

$$
Y_{p, K}=\left\{X \in[X] ; \sup _{n \in N} \frac{\left|X_{\Lambda_{2 n a / p}}\right|_{a / p \sqrt{2}}}{\log _{+} 2 n a / p}>K\right\}
$$

we have to prove that there exists $p(z)$ such that $\lim _{K \rightarrow \infty} \varrho_{z}\left(Y_{p(z), K}\right)=0$, when $\varrho_{z}$ is a solution of $(2.2)$.

Now let us introduce:

We have:

$$
Y_{p, K, N}=\left\{X \in[X] ; \frac{\left|X_{2 N a / p}\right|_{a / p \sqrt{2}}}{\log _{+} 2 N a / p}>K\right\} .
$$

$$
\varrho_{z}\left(Y_{p, K}\right) \leqq \sum_{N=1}^{\infty} \varrho_{z}\left(Y_{p, K, N}\right)
$$

so that we are led to seek an estimation of $\varrho_{z}\left(Y_{p, K, N}\right)$. To this purpose we remark that:

$$
Y_{p, K, N}=\bigcup_{r>K \log +2 N a / p}\left(\bigcup_{C \in C_{N, r}^{p}}[C]\right) .
$$

Let $C \in C_{N, r}^{p}$ and let us estimate $\varrho_{z}[C]$ when $\varrho_{z}$ is a solution of (2.2). We have:

$$
\varrho_{z}[C]=\left\{\text { Probability, with respect to } \varrho_{z} \text {, for } C \text { to be occupied. }\right\}
$$

As each cell of $C$ can be occupied by only one particle, we see that:

$$
\varrho_{z}[C]=\left\{\begin{array}{l}
\text { Probability of having exactly } r \text { particles } \\
\text { in the volume limited by } C
\end{array}\right\} .
$$

Therefore it follows immediately from (2.3) that:

$$
\varrho_{z}[C] \leqq\left(z a^{2} / p^{2}\right)^{r} .
$$

(We indicate here that a larger bound, but sufficient for the rest of the argument, can be obtained for states on $\mathfrak{U}$ having their correlation functions satisfying the bound $\varrho_{n}\left(q_{1}, \ldots, q_{n}\right) \leqq(z)^{n}$.) We remark that the bound (5.5) is independent on the shape of $C$, so that:

$$
\varrho_{z}\left(\bigcup_{C \in C_{N, r}^{p}}[C]\right) \leqq\left(z a^{2} / p^{2}\right)^{r} \operatorname{Card}\left(C_{N, r}^{p}\right) .
$$

An easy calculation leads to: $\operatorname{Card}\left(C_{N, r}^{p}\right) \leqq 4 N^{2}(8 p)^{r}$. From (5.4) we get:

$$
\varrho_{z}\left(Y_{p, K N}\right) \leqq \sum_{r>K} \sum_{\log +2 N a / p} 4 N^{2}\left(8 z a^{2} / p\right)^{r} .
$$
If we choose $p$ sufficiently large such that $\mu(p, z)=\frac{8 z a^{2}}{p}<1$ we shall
have:

$$
\varrho_{z}\left(Y_{p, K, N}\right) \leqq 4 N^{2}(\mu(p, z))^{K \log +2 N a / p} \times \frac{1}{1-\mu(p, z)} .
$$


Then from (5.4) we get:

$$
\varrho_{z}\left(Y_{p, K}\right) \leqq \frac{4}{1-\mu(p, z)} \sum_{N=1}^{\infty} N^{2}(\mu(p, z))^{K \log +2 N a / p} .
$$

The series in the right hand side is convergent for $K$ sufficiently large, provided $\mu(p, z)<1$, and its sum tends to 0 as $K$ tends to infinity. This proves the lemma and the proposition.

It results from the Propositions 3.4 and 5.1 that, if $\varrho_{z}$ is a solution of (2.2), there exists $\alpha(z)>0$ such that $\varrho_{z}^{t}$ is defined by (5.1) provided $|t|<\alpha(z)$. We now prove the time invariance of $\varrho_{z}$ during this time interval.

Proposition 5.3. For $\alpha(z)$ given by Proposition 5.1, we have: $\varrho_{z}^{t}=\varrho_{z}$, $\forall t,|t|<\alpha(z)$.

Proof. In view of Proposition 2.3, it sufficies to prove that for any bounded open $\Lambda \subset R^{2}$ and any $\varphi \in \mathfrak{U}_{\Lambda}$ we have:

$$
\int\left(\varphi \circ T^{t}\right) \varrho_{z}=\int \varphi \varrho_{z} \quad \text { if } \quad|t|<\alpha(z) .
$$

We set $\|\varphi\|=\sup _{X \in[X]}|\varphi(X)|$. From Lemma 5.2 we have:

so that:

$$
\begin{gathered}
\forall \varepsilon>0, \quad \exists K: \varrho_{z}\left([X] \backslash[X]_{\alpha(z), K}^{\prime}\right)<\frac{\varepsilon}{2\|\varphi\|} \\
\left|\int\left(\varphi \circ T^{t}\right) \varrho_{z}-\int_{[X]_{\alpha, K}^{\prime}}\left(\varphi \circ T^{t}\right) \varrho_{z}\right|<\varepsilon / 2 .
\end{gathered}
$$

As $\varphi$ is measurable in $\Lambda$, we may write $\varphi\left(T^{t}(X)\right)=\varphi\left(T^{t}(X)\right)_{\Lambda}$ and using Proposition 3.5, we see that:

$$
\exists l>0:\left(T^{t}(X)\right)_{\Lambda}=\left(\tilde{T}_{\Lambda_{l}}^{t}\left(X_{\Lambda_{l+2 a}}\right)\right)_{\Lambda}, \quad \forall X \in[X]_{\alpha(z), K}^{\prime}, \quad \forall t, \quad|t|<\alpha(z) .
$$

Hence:

$$
\exists l>0: \int_{[X]_{\alpha(z), K}^{\prime}}\left(\varphi \circ T^{t}\right) \varrho_{z}=\int_{[X]_{\alpha(z), K}^{\prime}}\left(\varphi \circ \tilde{T}_{\Lambda_{l}}^{t}\left(X_{\Lambda_{l}+2 a}\right)\right) \varphi_{z}, \forall t,|t|<\alpha(z) .
$$

We use (5.6) once more to get:

$$
\exists l>0:\left|\int_{[X]_{x, \mathrm{~K}}^{\prime}}\left(\varphi \circ T^{t}\right) \varrho_{z}-\int\left(\varphi \circ \tilde{T}_{\Lambda_{l}}^{t}\left(X_{\Lambda_{l}+2 a}\right)\right) \varrho_{z}\right|<\varepsilon / 2 .
$$

Adding (5.7) and (5.8) we finally have:

$$
\forall \varepsilon>0, \exists l>0: \mid \int\left(\varphi \circ T^{t}\right) \varrho_{z}-\int\left(\varphi \circ \tilde{T}_{\Lambda_{l}}^{t}\left(X_{\Lambda_{l+2 a}}\right) \varrho_{z} \mid<\varepsilon .\right.
$$

Now we remark that $\varphi \circ \tilde{T}_{\Lambda_{l}}^{t}\left(X_{A_{l+2 a}}\right)$ is measurable in the box $\Lambda_{l+2 a}$, so that we can apply the equilibrium equations (2.2) to get:

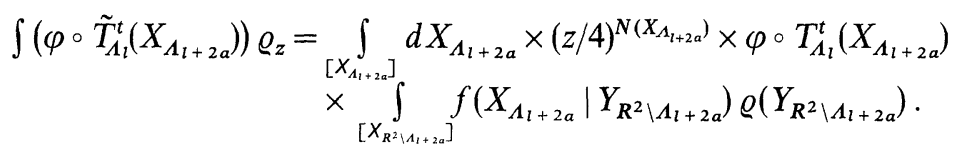

We note that $d X_{A_{l+2 a}}$ is invariant under $\tilde{T}_{A_{l}}^{t}$ for two reasons: first the particles lying inside $\Lambda_{l+2 a} \backslash \Lambda_{l}$ do not move, second for the particles 
lying inside $\Lambda_{l}$ we can apply the Liouville's theorem. It is also clear that:

$$
f\left(\tilde{T}_{\Lambda_{l}}^{t}\left(X_{\Lambda_{l+2 a}}\right) \mid Y_{R^{2} \backslash \Lambda_{l+2 a}}\right)=f\left(X_{\Lambda_{l+2 a}} \mid Y_{R^{2} \backslash \Lambda_{l+2 a}}\right) .
$$

This leads to:

$$
\int\left(\varphi \circ \tilde{T}_{A_{l}}^{t}\left(X_{A_{l+2 A}}\right)\right) \varrho_{z}=\int \varphi \circ \varrho_{z} .
$$

Therefore, combining this with (5.9):

$$
\forall \varepsilon>0, \quad\left|\int\left(\varphi \circ T^{t}\right) \varrho_{z}-\int \varphi \varrho_{z}\right|<\varepsilon, \quad \forall t, \quad|t|<\alpha(z) .
$$

This achieves the proof of the proposition.

It is now easy to achieve the programme mentionned at the beginning of this section. Let $\varrho_{z}$ be a solution of (2.2), we can choose an $\alpha(z)$ such that $\varrho_{z}[X]_{\alpha(z)}^{0}=1$ because $[X]_{\alpha(z)}^{\prime \prime} \subset[X]_{\alpha(z)}^{0}$. From the above proposition we have:

$$
\varrho_{z}\left(T^{t}[X]_{\alpha(z)}^{0}\right)=\varrho_{z}\left([X]_{(z)}^{0}\right)=1, \quad \forall t:|t|<\alpha(z) .
$$

Therefore $\varrho_{z}[X]_{\alpha(z)}^{1}=1$ and by induction on $n, \varrho_{z}[X]_{\alpha}=1$. The time invariance of $\varrho_{z}$ during any time interval is a consequence of the group property of $T^{t}$ on $[X]_{\alpha}$. We have then proved the following theorem:

Theorem 5.4. For a given activity $z$, there exists a Borel set $[X]_{\alpha} \subset[X]$ such that:

a) $\varrho_{z}[X]_{\alpha}=1$ for every solution $\varrho_{z}$ of (2.2).

b) $T^{t}: R \times[X]_{\alpha} \rightarrow[X]_{\alpha}$ is a Borel mapping and a one-parameter group of Borel isomorphisms on $[X]_{\alpha}$.

c) $\varrho_{z}$ is invariant under $T^{t}$.

We conclude this section with some remarks concerning the conservation of entropy. If $\varrho$ is a state on $\mathfrak{U}$ invariant under translation, we can define the entropy of $\varrho$ along the same lines as in Ref. [2]. Nevertheless there are many simplifications being due to the fact that the momentum space for each particle is compact in our case. In addition the particle number and the kinetic energy per unit volume is bounded in our case, so that following the argument developed in the propositions 7.1 and 7.2 of Ref. [2], it is easy to prove that any homogeneous state concentrated on some $[X]_{\alpha}$ has a constant entropy during the time evolution.

\section{§ 6. Remarks}

First we emphasize a weakness of our Theorem 5.4. In fact, we do not have a convenient criterion for a state to be concentrated on some $[X]_{\alpha}$. The Radon-Nikodym theorem allows to assert that every state of the form $\varphi \varrho_{z}$ where $\varphi \in L^{1}\left([X], \varrho_{z}\right)$ and $\varrho_{z}$ is a solution of (2.2) is concentrated on some $[X]_{\alpha}$. Such states are essentially inhomogeneous states and describe local perturbations from the equilibrium state $\varrho_{z}$. It remains to prove the existence of non trivial states, concentrated on 
some $[X]_{\alpha}$ but which are non-equilibrium homogeneous states. A case of interest is the state with the same correlations as $\varrho_{z}$ but with an anisotropic distribution in the momentum space. If $\varrho_{z}$ is homogeneous and ergodic with respect to translations (this is the case for small values of $z$ ) the following proposition gives an answer:

Proposition 6.1. Let $\varrho$ be a homogeneous state, ergodic with respect to translations, having correlation functions satisfying the bound $\varrho_{n}\left(q_{1}, \ldots, q_{n}\right)$ $\leqq z^{n}$, then there exists $\alpha>0$ such that $\varrho[X]_{\alpha}=1$.

Proof. Following the same lines as in the proof of Lemma 5.2 it can be shown that there exists $\alpha(z)$ such that $\varrho[X]_{\alpha}^{\prime \prime}=1$ for any $\alpha \leqq \alpha(z)$. We can choose $\alpha(z)$ such that $\varrho[X]_{\alpha(z)}^{0}=1$. Let us show that $\varrho\left(T^{t}[X]_{\alpha(z)}^{0}\right)=1$, $\forall t,|t| \leqq \alpha(z)$. In fact $[X]_{\alpha(z)}^{0}$ is invariant under translations, so that the same is valid for $T^{t}[X]_{\alpha(z)}^{0}$ if $|t|<\alpha(z)$. As $\varrho$ is ergodic with respect to translations we have: $\varrho\left(T^{t}[X]_{\alpha(z)}^{0}\right)=0$ or 1 . Now it is easy to show that $\varrho\left(T^{t}[X]_{\alpha(z)}^{0}\right)$ is a continuous function of $t$ if $|t|<\alpha(z)$. Thus $\varrho\left(T^{t}[X]_{\alpha(z)}^{0}\right)=1$ for any $|t|<\alpha(z)$, and $\varrho[X]_{\alpha(z)}^{1}=1$. One then prove by induction that $\varrho[X]_{\alpha(z)}=1$.

The results of this paper are to be taken as a preliminary step towards a study of the dynamical properties of the model. The main question to investigate is whether or not the dynamical system $([X]$, $\varrho_{z}, T^{t}$ ) is a $K$-System (at least for small values of $z$ ). But the answer to this question is a considerably more difficult problem.

Finally, we mention that there exists an analogue in three dimensions of the model: namely a system of hard cubes with only six directions of velocities. It is clear that all the results of this paper can be transposed into this case. We have then two models which should be good candidates to investigate the question of existence or non existence of transport coefficients with respect to the dimension of the system [8].

Acknowledgements. I am grateful to Pomeau, Robinson and Lanford for stimulating discussions and helpful suggestions.

\section{References}

1. Lanford, O. E.: Commun. math. Phys. 9, 169-191 (1968).

2. Lanford, O.E.: Commun math. Phys. 11, 257-292 (1969).

3. Sinai, Ya. G.: Funct. Anal. Appl., 6, 41-50 (1972) (in Russian).

4. de Pazzis, O.: Commun. math. Phys. 22, 121-132 (1971).

5. Ruelle, D.: Statistical Mechanics, New York: Benjamin 1969.

6. Ruelle, D.: Commun. math. Phys. 18, 127 (1970).

7. Lanford, O.E., Ruelle, D.: Commun. math. Phys. 13, 194 (1969).

8. Pomeau, Y.: Phys. Rev. A 3, 1174-1194 (1971).

\section{O. de Pazzis}

Département de Physique

Observatoire de Meudon

F-92 Meudon, France 